\title{
Necrotizing Fasciitis Arising From an Enterocutaneous Fistula in a Case of an Appendiceal Mucocele
}

\author{
Keun Young Kim, Won Cheol Park \\ Department of Surgery, Wonkwang University School of Medicine, Iksan, Korea
}

An appendiceal mucocele (AM) is a rare tumorous condition of the appendix. Many patients with AM are admitted to the hospital with abdominal pain or discomfort, and many cases are found incidentally. Although the rate of complications in patients with AM is very low, if left untreated, a mucocele may rupture and produce a potentially fatal entity known as pseudomyxoma peritonei. In this paper, we report a case of an 80-year-old man with necrotizing fasciitis arising from an enterocutaneous fistula caused by AM.

Keywords: Appendiceal neoplasms; Mucocele; Necrotizing fasciitis

\section{INTRODUCTION}

An appendiceal mucocele (AM) is a very rare appendiceal disease. The initial diagnosis is usually acute appendicitis, and an AM is often diagnosed during surgery or after surgical biopsy [13]. Although many reports have been published on AM, the occurrence rate of complications is very low. Prognosis is poor in some cases owing to pseudomyxoma peritonei induced by the ruptured mucocele. Here, we report a rare case of a patient with an AM who developed necrotizing fasciitis caused by the formation of an enterocutaneous fistula.

\section{CASE REPORT}

Two years ago, an 80-year-old man with intermittent abdominal discomfort in the right lower quadrant of the abdomen was admitted to our department via a local clinic. Although he complained of intermittent abdominal pain and diarrhea, the physical examination and blood test findings were normal. The patient

Received: December 10,2014 - Accepted: September 20, 2015

Correspondence to: Won Cheol Park, M.D.

Department of Surgery, Institute of Medical Science, Wonkwang University

School of Medicine, 460 lksan-daero, Iksan 54538, Korea

Tel: +82-63-859-1496, Fax: +82-63-855-2386

E-mail: parkwc@wonkwang.ac.kr

(C) 2015 The Korean Society of Coloproctology

This is an open-access article distributed under the terms of the Creative Commons Attribution NonCommercial License (http://creativecommons.org/licenses/by-nc/3.0) which permits unrestricted non-

commercial use, distribution, and reproduction in any medium, provided the original work is properly cited. had undergone an intestinal resection at the age of 35 from complications due to tuberculous enteritis. He was currently taking medication for hypertension. A physician in our department performed computed tomography (CT) because of a cystic lesion found within the right lower quadrant of the abdomen through ultrasonography at the referring clinic. The CT scan showed a 5-cm regular hypodense lesion near the cecum without enlarged lymph nodes (Fig. 1A). The radiologist indicated that the lesion resembled an AM, but was most likely terminal ileitis due to a postoperative adhesion or the tuberculous enteritis.

The patient did not come to hospital for checking the CT results. He then experienced chest pain and visited the Cardiology Department. Coronary angiography revealed angina pectoris, for which he was treated for 3 months. His cardiac problems were resolved, and he revisited the outpatient Gastroenterology Department. At that time, his physician recommended surgery, but he and his guardian refused because of his medical history of cardiac problems. They requested medication instead. For 1 year, the patient was regularly followed up by the outpatient Gastroenterology Department. We repeated the CT scan at 1 year and found that the mucocele had increased to $6 \mathrm{~cm}$ (Fig. 1B). Again, we recommended surgery, which the patient refused. Three months later, he was admitted to the Emergency Department with abdominal pain and a fever of $38.3^{\circ} \mathrm{C}$. His white blood cell (WBC) count was $15.8 \times 10^{3} / \mu \mathrm{L}$, and his C-reactive protein (CRP) level was elevated $(146 \mathrm{mg} / \mathrm{L})$. On physical examination, we found a 7 -cm cavity in the right lower quadrant that showed tenderness, heat, and erythema. We performed another CT to evaluate the patient for an intraperitoneal disease such as an abscess or pseu- 

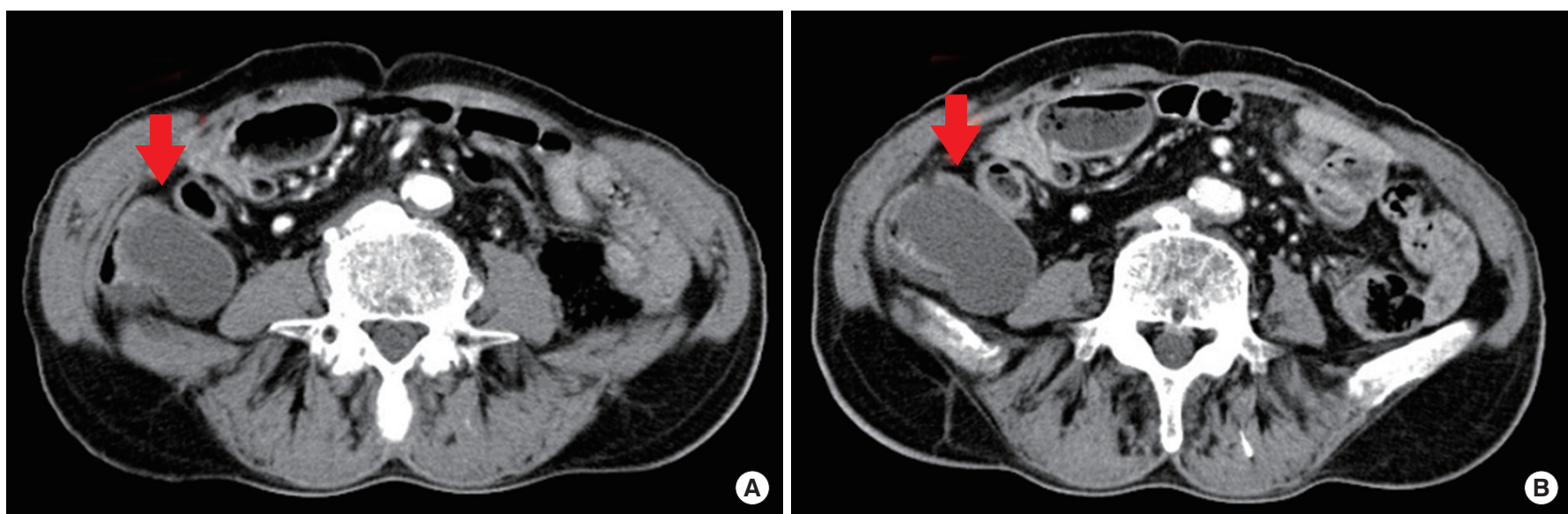

Fig. 1. (A) An initial computed tomography scan shows a 5-cm hypodense lesion near the cecum without enlarged lymph nodes (arrow). (B) A computed tomography scan taken on 1 year later revealed that the mucocele was $6 \mathrm{~cm}$ (arrow).
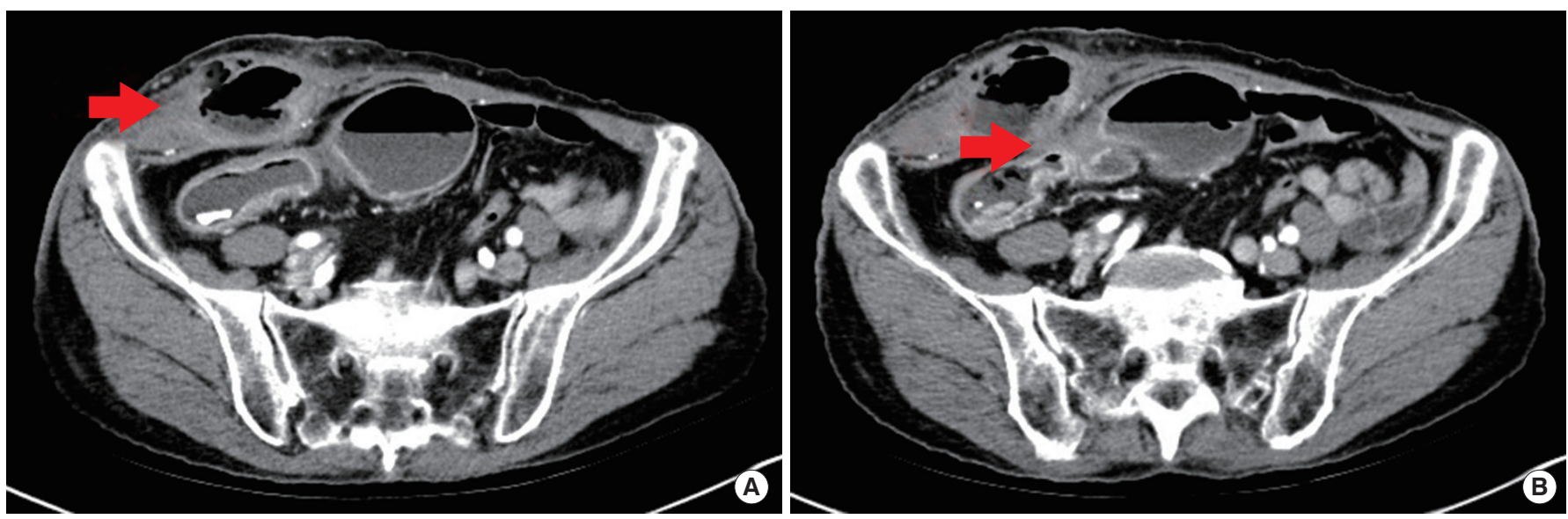

Fig. 2. (A) Computed tomography scan taken three months later showed an abscess cavity with an air-fluid level in the right lower quadrant (arrow). (B) The computed tomography scan showed an enterocutaneous fistula tract (arrow).

domyxoma peritonei. The CT showed a 7-cm abscess cavity on the right abdominal wall with an air-fluid level (Fig. 2), as well as a fistular tract. Fortunately, there was no evidence of pseudomyxoma peritonei.

We performed an emergency operation and found a $6 \times 6-\mathrm{cm}$ abscess on the rectus muscle with transversalis fascial necrosis. We then performed wide debridement (fascia and muscle) and opened the wound. The pus within the wound was dark brown and had a foul odor; therefore, we performed a bacterial culture. We performed another wide debridement of the fascia and muscle, after which the wound healed. The patient's WBC count and CRP level normalized. The initial bacterial culture revealed Escherichia coli and Klebsiella pneumoniae. After the operation, repeated culture results showed Pseudomonas aeruginosa and methicillin-resistant Staphylococcus aureus. One week later, however, feces were seen in the wound, and we found a fistula open- ing. We decided to operate for the tentative diagnosis and treatment. Two weeks later, we performed a diagnostic laparoscopy and found that the cecum and distal ileum were abutting the abdominal wall below the wound. The small bowel and transverse colon were severely dilated and were adhering to each other. A small mucinous lesion was found. Fortunately, no evidence of pseudomyxoma peritonei was found (Fig. 3).

We performed a right hemicolectomy with lymphatic dissection for the detection of a hidden malignancy such as mucinous cystadenocarcinoma as well as a fistulectomy (Fig. 4). The wound was closed at the peritoneal and posterior fascial layers while the skin and anterior fascia remained open for dressings. After the colon resection, we performed debridement of the fascia and covered the area with dressings for 3 weeks. One month later, the wound was nearly healed; therefore, we performed wound repair with prosthetic mesh. The pathological report indicated a low-grade 


\section{Coloproctology}

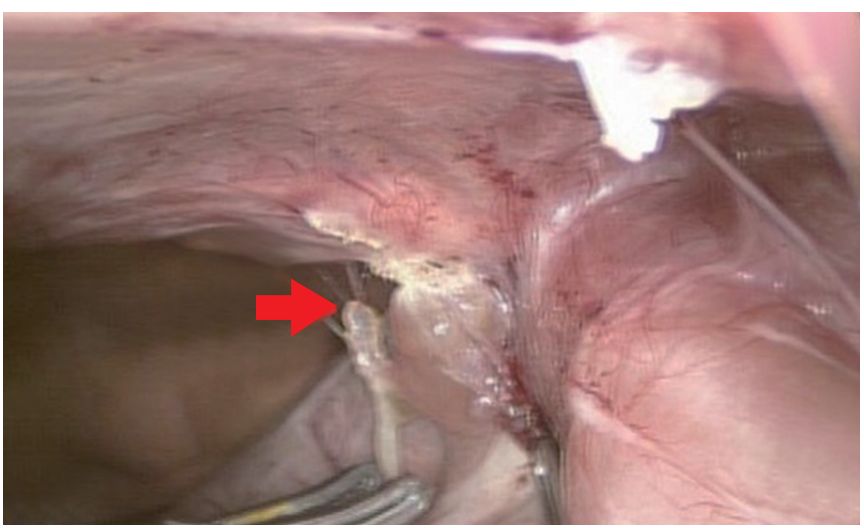

Fig. 3. Laparoscopy showed that the cecum and distal ileum had adhered to the abdominal wall and that a small mucinous cystic lesion (arrow) was visible near the cecum.

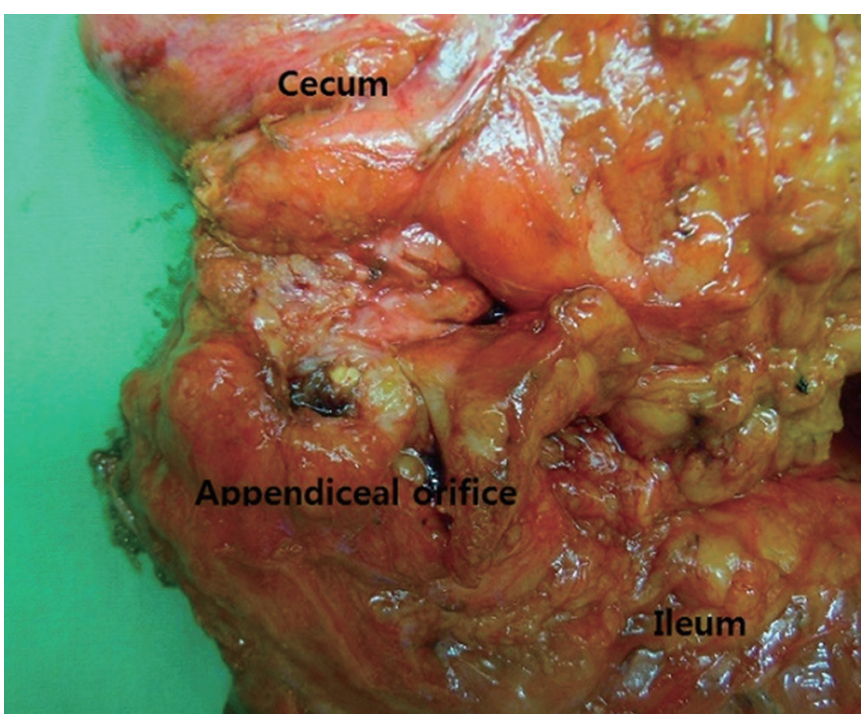

Fig. 4. The specimen after an extended right hemicolectomy.

mucinous cystadenoma and no metastatic lymph nodes (Fig. 5). The patient is now completely healed and has not experienced recurrence.

\section{DISCUSSION}

An AM causes mucinous distention of the appendiceal lumen. The pathological entity includes a retention cyst, mucosal hyperplasia, cystadenoma, and cystadenocarcinoma. Most cases of AM are benign. A mucinous cystadenocarcinoma of the appendix comprises a very small portion of all gastrointestinal malignancies. However, the prognosis of an appendiceal cystadenocarcinoma is very poor because there is no proper treatment at relapse beyond surgery. As such, providing an accurate diagnosis and proper treatment for AM early in the process is important [1-3].

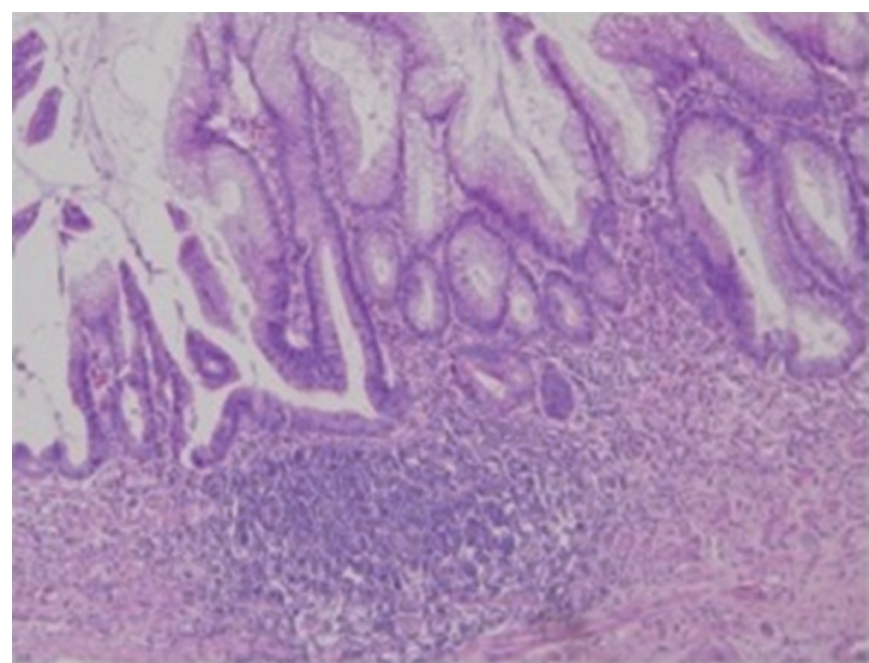

Fig. 5. Pathological analysis indicated a low-grade mucinous neoplasm within the appendix $(\mathrm{H} \& \mathrm{E}, \times 100)$.

The prevalence of AM is not well known because many patients are asymptomatic. Symptomatic lesions are more commonly associated with complications or malignant conditions than asymptomatic ones are. In cases of complicated AM, malignancy must be considered. In cases of a huge mucocele such as in this case, perforation into the abdominal cavity may occur or an abscess may develop. Moreover, for these patients, an abscess in the abdominal wall may form a fistula within three months. The mechanism of fistula formation in patients with an AM is unknown. Perhaps chronic inflammation places pressure on the abdominal wall and creates an enterocutaneous tract; however, no study has examined the incidence of complications related to an AM. Usually, a mucocele $>2 \mathrm{~cm}$ that contains a solid nodule or a symptomatic mucocele (obstruction or pain) must be resected to prevent complications and the possibility of cancer. In many cases of surgical resection for an unruptured AM, no complications or pseudomyxoma peritonei are reported [1, 3-6].

We reviewed our hospital's records and found that a total of 23 patients had had a histological diagnosis of an AM (Table 1). Eight of these patients were admitted to the Emergency Department while the remaining 15 patients were admitted through outpatient departments. Three cases were incidentally found during screening for other diseases such as cervical cancer $(n=2)$ and colon cancer $(n=1)$. As with several other reported diseases, an AM is more prevalent in women [1]. Thirteen patients had symptoms of right lower quadrant abdominal pain or discomfort, three had nonspecific symptoms such as indigestion, and the other seven had no symptoms. The WBC counts were elevated in seven patients while they were normal in the rest. None of the patients had a fever. All 23 patients underwent preoperative abdominal $\mathrm{CT}$, and the AM was diagnosed preoperatively in 21 of the 23 . The other two patients were initially diagnosed with a periappen- 
Table 1. Clinicopathologic results of appendiceal mucocele at our hospital

\begin{tabular}{lc}
\hline Variable & No. of cases \\
\hline Patients & 23 \\
Sex & \\
Male:female & $9: 14$ \\
Age (yr) & \\
\hline $40-49$ & 3 \\
\hline $50-59$ & 4 \\
$60-69$ & 2 \\
\hline $70-79$ & 4 \\
\hline $80-89$ & 8 \\
\hline $90-99$ & 2 \\
Operation method & 8 \\
\hline Appendectomy & 13 \\
Cecectomy & 1 \\
\hline Ileocectomy & 1 \\
\hline Right hemicolectomy & 20 \\
\hline Pathology results & 1 \\
\hline Mucosal hyperplasia & 2 \\
\hline Mucinous cystadenoma & \\
\hline Mucinous cystadenocarcinoma & \\
\hline
\end{tabular}

diceal abscess and treated with external drainage. Three months later, we performed a laparoscopic cecectomy. The biopsy result was a mucinous cystadenoma. The patients were 43-92 years of age. Seven were in their 40 s or 50 s while the ages of the remaining patients were $>60$ years. The size of the AM varied widely from $2.2-6 \mathrm{~cm}$. Only one patient had complications $[3,7]$.

A CT scan was taken in all cases, and a preoperative diagnosis was possible in all but two cases. Our preoperative diagnostic rate is higher than that of other reports in which patients underwent ultrasonography only. In the diagnosis of an AM, the CT scan is superior to ultrasonography. If a patient has an unusual abdominal symptom, we should consider doing a CT scan. The two patients suspected of having a periappendiceal abscess underwent percutaneous drainage, followed by an interval appendectomy, and the diagnosis was later confirmed in the pathologic report. The inflammation associated with an AM is easily confused with acute appendicitis; therefore, significant clinical attention is required. The AM in each patient was $>2 \mathrm{~cm}$; however, complications were seen in only patient. Six patients had increased WBC and CRP levels while only one patient had a fever [3].

The possibility of colon cancer in elderly patients with abdominal symptoms should be considered, and we believe the same about an AM. Because an AM is accompanied by inflammation, it is easily confused with acute appendicitis. In particular, we must carefully choose procedures such as percutaneous drainage. Especially, a malignant AM is present in $95 \%$ of the pseudomyxoma peritonei cases in some reports. A cautious approach is needed for a periappendiceal abscess in the elderly. Although the relationship between the risk of complications and the size of the AM remains unclear, we recommend early surgery for patients who have symptoms or significant inflammation [8].

Most AMs are found incidentally in asymptomatic cases or are found to be due to acute appendicitis, such as inflammatory disease. However, according to several reports, AMs are seen to be accompanied by serious complications such as in this case. An AM may cause intestinal bleeding accompanied by anemia and may lead to intestinal obstruction caused by extraluminal compression [9]. It can also be caused by an acute abdomen, such as intestinal obstruction by volvulus or torsion $[10,11]$. The problem is that these emergency situations will increase the possibility of AM rupture, which may eventually lead to fatal complication of pseudomyxoma peritonei. An interesting point is that no obvious relationship has been found between the tumor size and the incidence of complications. Therefore, if the AM is found, immediate surgery will yield good results with a good prognosis for the patient [10-12].

Most appendiceal diseases can be ultrasonically diagnosed. According to reports from other centers, the accurate diagnostic rate of an AM before surgery is $18 \%-40 \%$. Although appendiceal tumors are rare, abdominal tomography is necessary in a patient with long-term symptoms who is older than 40 years of age and has normal blood test results but tenderness in the right lower quadrant or suprapubic area [1-3]. If an AM is preoperatively diagnosed, the existence of an accompanying tumor must be investigated. If a tumor exists, a complete resection is needed to better understand the extent of the mucocele via colonoscopy. Ensuring an adequate margin on resection during surgery in the diagnosis of acute appendicitis and performing the operation at an early stage if a patient is symptomatic or the AM is large is important $[6,12]$.

\section{CONFLICT OF INTEREST}

No potential conflict of interest relevant to this article was reported.

\section{ACKNOWLEDGMENTS}

This paper was supported by Wonkwang University 2014.

\section{REFERENCES}

1. Lim YC, Choi DH, Cho HR, Ko BK, Nam CW, Nah YW, et al. Appendiceal mucocele. J Korean Soc Coloproctol 2004;20:339-43.

2. Park SY, Lee JN, Chung M, Lee UG, Lee YD, Oho JH, et al. Appendiceal mucocele. J Korean Surg Soc 2007;72:157-61.

3. Cho JB, Cho HJ, Shin OR, Kim KH, Ahn CH, Kim JS, et al. Clini- 
cal findings of appendiceal mucocele. J Korean Surg Soc 2008;74: 429-35.

4. Karakaya K, Barut F, Emre AU, Ucan HB, Cakmak GK, Irkorucu $\mathrm{O}$, et al. Appendiceal mucocele: case reports and review of current literature. World J Gastroenterol 2008;14:2280-3.

5. Chang EY, Park JH, Rhee JA, Cho HC, Gu MJ. Clinical analysis and laparoscopic surgical experience for an appendiceal mucocele. J Korean Soc Endosc Laprosc Surg 2009;12:31-6.

6. Johnson MA, Jyotibasu D, Ravichandran P, Jeswanth S, Kannan DG, Surendran R. Retention mucocele of distal viable remnant tip of appendix: an unusually rare late surgical complication following incomplete appendectomy. World J Gastroenterol 2006;12: 489-92.

7. Rampone B, Roviello F, Marrelli D, Pinto E. Giant appendiceal mucocele: report of a case and brief review. World J Gastroenterol 2005;11:4761-3.
8. Lakatos PL, Gyori G, Halasz J, Fuszek P, Papp J, Jaray B, et al. Mucocele of the appendix: an unusual cause of lower abdominal pain in a patient with ulcerative colitis: a case report and review of literature. World J Gastroenterol 2005;11:457-9.

9. Pitiakoudis M, Tsaroucha AK, Mimidis K, Polychronidis A, Minopoulos G, Simopoulos C. Mucocele of the appendix: a report of five cases. Tech Coloproctol 2004;8:109-12.

10. Hamada T, Kosaka K, Shigeoka N, Hashimoto Y, Yamauchi M, Cho $\mathrm{S}$, et al. Torsion of the appendix secondary to appendiceal mucocele: gray scale and contrast-enhanced sonographic findings. J Ultrasound Med 2007;26:111-5.

11. Rudloff U, Malhotra S. Volvulus of an appendiceal mucocele: report of a case. Surg Today 2007;37:514-7.

12. Zagrodnik DF 2nd, Rose DM. Mucinous cystadenoma of the appendix: diagnosis, surgical management, and follow-up. Curr Surg 2003;60:341-3. 